\title{
Optimal Parameters of Moving Average Control Chart
}

\author{
Yupaporn Areepong
}

\begin{abstract}
The objective of this paper is to study Statistical Process Control (SPC) with a Moving Average control chart (MA) for monitoring the non-conforming product. The characteristic of control chart is Average Run Length (ARL) which is the average number of samples taken before an action signal is given. The ARL should be sufficiently large while the process is still in-control and the Average Delay time (AD) (mean delay of true alarm times) should be small when the process goes out-of-control. The explicit formulae of ARL and AD for MA-based are presented when observations are from binomial distribution. In particular, the explicit analytical formulas for evaluating ARL and AD be able to get a set of optimal parameters which depend on a width of the moving average $(w)$ and width of control limit $(H)$ for designing MA chart with minimum of AD.
\end{abstract}

Index Terms-Optimal parameters, Average Run Length, Average Delay time, Moving Average control chart, Nonconforming.

\section{INTRODUCTION}

In the past few decades, cumulative sum (CUSUM) and exponentially weighted moving average (EWMA) charts have been proposed as good alternatives to the Shewhart chart for detecting small shifts. The CUSUM chart was initially presented by Page [1]. It has been shown that CUSUM chart is asymptotically optimal under minimax type criteria (see, e.g., Lorden [2]). The EWMA chart was initially introduced by Roberts [3]. It is a very flexible and effective chart for detecting small changes and has the advantage of showing robustness to non-normality (Borror et al. [4]; Stoumbos and Reynolds [5]). When the quality characteristic cannot be measured on a continuous scale, as, for example, in counting the number of defective products or the number of nonconformities in a production process, an attribute control chart must be used. Commonly-used attribute control charts are $\mathrm{p}, \mathrm{np}, \mathrm{c}$, and $\mathrm{u}$ charts. EWMA and CUSUM methods for attribute data have also been applied to discrete processes (see, e.g., Alwan [6]). Recently, the Moving Average control chart (MA) has been introduced for both continuous and discrete processes (see, e.g., Alwan [6]). Michael and Khoo [7] have studied the MA chart for monitoring the non-conforming or defective fraction in discrete processes.

The characteristic of control chart is Average Run Length (ARL) which is the average number of samples taken before an action signal is given. The ARL should be sufficiently large while the process is still in-control and the Average Delay time (AD) (mean delay of true alarm times) should be

Manuscript received May 18, 2012; revised June 16, 2012

Yupaporn Areepong is with the Department of Applied Statistics, Faculty of Applied Science, King Mongkut's University of Technology North Bangkok, Bangkok 10800, Thailand. small when the process goes out-of-control. Many methods for evaluating the ARL and AD for control charts have been studied in the literature. A simple approach that is often used to test other methods is Monte Carlo (MC) simulation. MC is simple to program and is convenient for controlling and testing accuracy of analytical approximations. However, MC is usually based on a large number of sample trajectories so it is very time consuming. Markov Chain Approach (MCA) is considered as a popular technique. It is based on approximation of Markov Chains by using matrix inversions. Although there are at present no theoretical results on accuracy of this procedure, the results have been tested by direct comparison with MC simulations. Integral Equation (IE) is the most advanced method currently available. However, the results for ARL and AD usually cannot be obtained analytically and intensive programming or specialized software is required to obtain numerical results. Recently, explicit formulas for evaluation ARL have been presented. Areepong [8] derived analytical formulas for $\mathrm{ARL}$ and $\mathrm{AD}$ for MA chart when observations are from a binomial distribution. In this paper, using the explicit formulas we have been able to provide the tables for the optimal width of the moving average and width of control limit $(H)$ with minimum $\mathrm{AD}$ for MA chart for binomial distribution.

\section{CONTROl Charts AND TheIR Properties}

In this paper, SPC charts are considered under the assumption that sequential observations $X_{1}, X_{2}, \ldots$ of some process are identical, independently distributed random variables with a distribution function $F(x, n, p)$, where $n$ is the total number of observations and $p$ is a control parameter. It is assumed that $p=p_{0}$ while the process is in-control and $p=p_{1}>p_{0}$ when the process goes out-of-control. It is assumed that there is a changepoint time $\theta \leq \infty$ at which the parameter changes from $p=p_{0}$ to $p=p_{1}$. Note that $\theta=\infty$ means that the process always remains in the in-control state. All popular charts, such as Shewhart, CUSUM and EWMA are based on some function of parameter values that is used as a criterion for a process to go "out-of-control" if this function value goes above an upper control limit (UCL) or below a lower control limit (LCL). The minimum time required for a chart to signal out-of-control is defined as the stopping (alarm) time $\tau$.

Let $E_{\theta}($.$) denote the expectation that the change-point$ from $p=p_{0}$ to $p=p_{1}$ for a distribution function 
$F(x, n, p)$ occurs at time $\theta$, where $\theta \leq \infty$. In the literature on quality control the quantity $E_{\infty}(\tau)$ is called the Average Run Length (ARL) of the chart for the given process.

A typical condition imposed on an ARL is that:

$$
A R L=E_{\infty}(\tau)=T
$$

where $T$ is given (usually large). For given distribution function and chart, this condition then determines choices for the UCL and LCL.

A typical definition of the AD is that

$$
A D=E_{1}(\tau \mid \tau \geq 1),
$$

For example, that the change point occurs at $\theta=1$. One could expect that a sequential control chart has a near optimal performance if its $\mathrm{AD}$ is close to a minimal value.

For an np chart, let observations $X_{1}, X_{2}, \ldots, X_{m}$ be i.i.d random variables with binomial distribution, where $X_{i}$ the number of nonconforming is items in sample $i$ of $m$ samples of size $n$. For these observations, the $3 \sigma$ upper and lower control limits at $n \bar{p}$ after $m$ samples are defined by

$U C L=n \bar{p}+3 \sqrt{n \bar{p}(1-\bar{p})}, \quad L C L=n \bar{p}-3 \sqrt{n \bar{p}(1-\bar{p})}$

where $\bar{p}$ is estimated by $\bar{p}=\frac{\sum_{i=1}^{m} X_{i}}{n m}$.

The alarm time for the $n p$ chart is given by:

$$
\tau=\inf \left\{i>0: X_{i}>U C L \text { or } X_{i}<L C L\right\} .
$$

A Moving Average control chart is defined by the following statistics:

$$
\begin{gathered}
M_{i}=\frac{X_{i}+X_{i-1}+\ldots+X_{i-w+1}}{w}=\frac{\sum_{j=i-w+1}^{i} X_{j}}{w}, \mathrm{i} \geq \mathrm{w} \text { and } \\
M_{i}=\frac{X_{i}+X_{i-1}+\ldots+X_{1}}{i}=\frac{\sum_{j=1}^{i} X_{j}}{i}, 1 \leq \mathrm{i}<\mathrm{w} .
\end{gathered}
$$

where $w$ is the width of the moving average chart.

For period $i \geq w$, the $3 \sigma$ upper and lower control limits are given

$$
\begin{gathered}
U C L=n p+3 \sqrt{\frac{n p(1-p)}{w}} \\
L C L=n p-3 \sqrt{\frac{n p(1-p)}{w}}
\end{gathered}
$$

and for periods $i<w, \sqrt{\frac{n p(1-p)}{w}}$ is replaced with $\sqrt{\frac{n p(1-p)}{i}}$.

The alarm time for the MA procedure is given by

$$
\tau=\inf \left\{i>0: M_{i}>U C L \text { or } M_{i}<L C L\right\}
$$

\section{A. Optimal Parameters for Binomial MA Chart}

The ARL values of a Moving Average control chart can be derived as follows:

Let $A R L=n$, then

$$
\begin{aligned}
& \frac{1}{A R L}=P(\text { out }- \text { of }- \text { control signal at time } i<w)+\left[\frac{n-(w-1)}{n}\right] P(\text { out }- \text { of }- \text { control signal at time } i \geq w) \\
& =\frac{1}{n}\left\{\sum_{i=1}^{w-1}\left[P\left(\frac{\sum_{j=1}^{i} n p_{j}}{i}>U C L_{i}\right)+P\left(\frac{\sum_{j=1}^{i} n p_{j}}{i}<L C L_{i}\right)\right]\right\}+\left[\frac{n-(w-1)}{n}\right]\left[P\left(\frac{1}{w} \sum_{j=i-w+1}^{i} n p_{j}>U C L_{w}\right)+P\left(\frac{1}{w} \sum_{j=i-w+1}^{i} n p_{j}<L C L_{w}\right)\right]
\end{aligned}
$$

then

$$
\begin{aligned}
& \frac{1}{A R L}=\frac{1}{n}\left\{\sum _ { i = 1 } ^ { w - 1 } \left[P \left(Z_{1}>\frac{n p_{0}+3 \sqrt{\frac{n p_{0}\left(1-p_{0}\right)}{i}}-n p}{\left.\left.\left.\sqrt{\frac{n p(1-p)}{i}}\right)+P\left(Z_{1}<\frac{n p_{0}-3 \sqrt{\frac{n p_{0}\left(1-p_{0}\right)}{i}}-n p}{\sqrt{\frac{n p(1-p)}{i}}}\right)\right]\right\}}\right.\right.\right. \\
&+\left[\frac{n-(w-1)}{n}\right]\left[P\left(Z_{2}>\frac{n p_{0}+3 \sqrt{\frac{n p_{0}\left(1-p_{0}\right)}{w}}-n p}{\sqrt{\frac{n p(1-p)}{w}}}\right)+P\left(Z_{2}<\frac{n p_{0}-3 \sqrt{\frac{n p_{0}\left(1-p_{0}\right)}{w}}-n p}{\sqrt{\frac{n p(1-p)}{w}}}\right)\right]
\end{aligned}
$$

As mentioned above, the value of the parameter $p$ is equal to $p_{0}$ when the process is in-control. Therefore, substituting $p=p_{0}$ into Equation (1), the formula for the desired ARL can be obtained by replacing 3 by width of control $(H)$. Then, the

$$
\begin{aligned}
& A R L= \\
& \left(1-\sum_{i=1}^{w-1}\left[P\left(Z_{1}>H\right)+P\left(Z_{1}<-H\right)\right]\right)\left[P\left(Z_{2}>H\right)+P\left(Z_{2}<-H\right)\right]^{-1} \\
& +(w-1)
\end{aligned}
$$

When the process is out-of-control, the value of the 
parameter $p$ in equation (1) will be $p=p_{1}$. The formula

written as follows:

for $\mathrm{AD}$ for a width of control limit $H$, can therefore be

$$
\begin{gathered}
A D=\left(1-\sum_{i=1}^{w-1}\left[P\left(Z_{1}>\frac{n p_{0}+H \sqrt{\frac{n p_{0}\left(1-p_{0}\right)}{i}}-n p_{1}}{\sqrt{\frac{n p_{1}\left(1-p_{1}\right)}{i}}}\right)+P\left(Z_{1}<\frac{n p_{0}-H \sqrt{\frac{n p_{0}\left(1-p_{0}\right)}{i}}-n p_{1}}{\sqrt{\frac{n p_{1}\left(1-p_{1}\right)}{i}}}\right)\right]\right. \\
\times\left[P\left(Z_{2}>\frac{n p_{0}+H \sqrt{\frac{n p_{0}\left(1-p_{0}\right)}{w}}-n p_{1}}{\sqrt{\frac{n p_{1}\left(1-p_{1}\right)}{w}}}\right)+P\left(Z_{2}<\frac{n p_{0}-H \sqrt{\frac{n p_{0}\left(1-p_{0}\right)}{w}}-n p_{1}}{\sqrt{\frac{n p_{1}\left(1-p_{1}\right)}{w}}}\right]^{-1}+(w-1) .\right.
\end{gathered}
$$

We first describe a procedure for obtaining optimal designs for binomial MA chart. The criterion used for choosing optimal values for is the width of the moving average chart $(w)$ and boundary parameter $(H)$ is minimization of $\mathrm{AD}$ for a given in-control parameter value $p_{0}=0.01, \mathrm{ARL}=\mathrm{T}$ and a given out-of-control parameter value $\left(p=p_{1}\right)$. We compute optimal $(w, H)$ values for $\mathrm{T}=370.4$ and 500 and magnitudes of change. Table of the optimal parameters values are shown in Table 2.

\section{B. The Numerical Procedure for Obtaining Optimal Parameters For Ma Designs}

1) Select an acceptable in-control value of ARL and decide on the change parameter value $\left(p_{0}\right)$ for an out-ofcontrol state.

2) For given $p_{0}$ and $\mathrm{T}$, find optimal values of $w$ and $H$ to minimize the $\mathrm{AD}$ values given by equation 3 subject to the constraint that $\mathrm{ARL}=\mathrm{T}$ in Equation 2, i.e. $w$ and $H$ are solutions of the optimality problem.

\section{Numerical Results}

In this section, the numerical results for ARL and $\mathrm{AD}$ for an MA chart were calculated from Equation (2) and Equation (3). Table 1 shows a comparison of these MA chart values with values obtained for np chart by simulation. The parameter values for MA and np charts were chosen by setting the desired ARL $=370$, the value of the in-control parameter $p_{0}=0.01$ and the out-of-control parameter $p_{1} \subseteq[0.013 ; 0.03] . \quad$ The MA chart shows a better performance than $\mathrm{np}$ from its in-control value $p_{0}=0.01$ to its out-of-control value $p_{1}$. Note that, calculations with the explicit formulas in Equation (2) and (3) are simple and very fast with computational times of less than 1 second.

\begin{tabular}{|c|c|c|c|c|c|c|c|c|c|c|c|}
\hline \multirow[t]{2}{*}{$\mathrm{n}$} & \multirow[t]{2}{*}{$p$} & \multicolumn{10}{|c|}{ MA } \\
\hline & & $\mathcal{W}=1$ & $w=2$ & $w=3$ & $w=4$ & $w=5$ & $w=6$ & $w=7$ & $w=8$ & $w=9$ & $w=10$ \\
\hline \multirow[t]{9}{*}{500} & 0.01 & 370.39 & 370.39 & 370.39 & 370.39 & 370.39 & 370.39 & 370.39 & 370.39 & 370.39 & 370.39 \\
\hline & 0.013 & 42.274 & 27.911 & 19.489 & 15.091 & 12.582 & 11.096 & 10.218 & 9.725 & 9.489 & 9.428 \\
\hline & 0.015 & 15.961 & 8.550 & 6.187 & 5.301 & 5.014 & 5.012 & 5.155 & 5.376 & 5.636 & 5.916 \\
\hline & 0.017 & 7.3387 & 4.136 & 3.413 & 3.316 & 3.431 & 3.619 & 3.8246 & 4.019 & 4.192 & 4.339 \\
\hline & 0.02 & 3.3733 & 2.278 & 2.207 & 2.317 & 2.443 & 2.543 & 2.611 & 2.653 & 2.678 & 2.692 \\
\hline & 0.023 & 2.0865 & 1.673 & 1.723 & 1.797 & 1.841 & 1.862 & 1.871 & 1.874 & 1.875 & 1.875 \\
\hline & 0.025 & 1.6850 & 1.467 & 1.519 & 1.559 & 1.575 & 1.580 & 1.582 & 1.582 & 1.582 & 1.582 \\
\hline & 0.027 & 1.4435 & 1.330 & 1.369 & 1.387 & 1.391 & 1.392 & 1.393 & 1.393 & 1.393 & 1.393 \\
\hline & 0.03 & 1.2371 & 1.196 & 1.214 & 1.219 & 1.219 & 1.219 & 1.219 & 1.219 & 1.219 & 1.219 \\
\hline
\end{tabular}

TABLE I: COMPARISON OF ARL AND AD FROM PROPOSED FORMULAS WITH NP CHART FOR GIVEN ARL=370

The numerical results in terms of optimal width of the moving average $(w)$, optimal width of control limit $(H)$ and minimal $\mathrm{AD}\left(A D^{*}\right)$ for $\mathrm{ARL}=370$ and 500 are shown in Table 2. For example, if we want to detect a parameter change from $p=0.01$ to $p=0.02$ and the ARL value is 370 then the optimality procedure given above will give optimal parameter values $w=3$ and $H=3$. On substituting the values for $p, w$ and $H$ into Equation 3 we obtain an optimal $\mathrm{AD}$ value $=2.207$. As shown in Table 1 and 2 the use of the suggested explicit formulas for ARL and AD for MA chart can greatly reduce the computation times, and are useful to practitioners especially finding optimal parameters of MA chart. 
TABLE II: OPTIMAL DESIGN PARAMETERS AND AD FOR BINOMIAL MA CHART

\begin{tabular}{|c|c|c|c|}
\hline \multicolumn{4}{|c|}{$\mathrm{T}=370$} \\
\hline$p$ & $w$ & $H$ & $\min \mathrm{AD}$ \\
\hline 0.013 & 10 & 3 & 9.4284 \\
\hline 0.015 & 6 & 3 & 5.0122 \\
\hline 0.017 & 4 & 3 & 3.3161 \\
\hline 0.02 & 3 & 3 & 2.2078 \\
\hline 0.025 & 2 & 3 & 1.4673 \\
\hline 0.03 & 2 & 3 & 1.1966 \\
\hline \multicolumn{4}{|c|}{$\mathrm{T}=500$} \\
\hline$p$ & $w$ & $H$ & $\min \mathrm{AD}$ \\
\hline 0.013 & 7 & 3.0905 & 5.3671 \\
\hline 0.015 & 7 & 3.0905 & 3.9494 \\
\hline 0.017 & 7 & 3.0905 & 2.7018 \\
\hline 0.02 & 3 & 3.0905 & 2.2803 \\
\hline 0.025 & 2 & 3.0905 & 1.5004 \\
\hline 0.03 & 2 & 3.0905 & 1.2124 \\
\hline
\end{tabular}

\section{ACKNOWLEDGEMENTS}

The author would like to express gratitude to Faculty of Science, King Mongkut's University of Technology, North Bangkok, Thailand for a supporting research grant.

\section{REFERENCES}

[1] E. S. Page, "Continuous inspection schemes," Biometrika, vol. 41, pp. 100-114, 1954.

[2] G. Lorden, "Procedures for reacting to a change in distribution," Annals of Mathematical Statistics, vol. 42, pp. 1897-1908, 1971.

[3] S. W. Roberts, "Control chart tests based on geometric moving average," Technometrics, vol. 1, pp. 239-250, 1959.

[4] C. M. Borror, D. C. Montgomery, and G. C. Runger, "Robustness of the EWMA Control Chart to Non-normality," Journal of Quality Technology, vol. 31, pp. 309-316, 1999.

[5] Z. G. Stoumbos and M. R. Reynolds, "Robustness to non-normality and autocorrelation of individuals control charts for monitoring the process mean and variance," Journal of Statistical Computation and Simulation, vol. 66, pp. 145-187, 2000.

[6] L. C. Alwan. Statistical Process Analysis, McGraw-Hill: New York, 1980.

[7] B. C. Michael and Khoo, "A Moving Average Control Chart for Monitoring the Fraction Non- conforming," International Journal of Quality and Reliability Engineering, vol. 20, pp. 617-635, 2004.

[8] Y. Areepong, "Explicit Formulas of Average Run Length for a Moving Average Control Chart for Monitoring the Number of Defective Products," International Journal of Pure and Applied mathematics. 